\title{
Free recall of categorially related list items over long retention intervals
}

\author{
DAVID L. HORTON \\ University of Maryland, College Park, Maryland 20740 \\ and \\ CHARLES N. COFER \\ Pennsylvania State University, University Park, Pennsylvania 16800
}

\begin{abstract}
Immediate and delayed (7, 14, and 28 days) free recalls of 40 -item lists were studied. The lists consisted either of high- or low-frequency associates of the category names and were presented in either blocked or random order. The delay groups did not differ at immediate recall, but item recall decreased with time up to 14 days. Categorial intrusions increased with delay. List organization as measured by repetitions declined with delay but not as measured by ratio of repetition. The findings are interpreted in terms of loss of knowledge of the list membership of categorial items with time.
\end{abstract}

Aside from two reports by Brand (1956; Brand \& Woods, 1958), there has been little work on the free recall of lists of words over substantial retention intervals. In Brand's studies, recalls were obtained at intervals of 1,2 , and 3 weeks following presentation of a list of items from the categories of the Allport-Vernon-Lindzey Study of Values. In these studies, there was an increase in the number of categorial intrusions over the retention interval. However, the value of these results is lessened by certain limitations of method; further, work on free recall has not usually employed value related materials. Consequently, it has seemed worthwhile to repeat Brand's experiments with more standard materials and under better controlled conditions.

In free recall of word lists, two findings lend further interest to the study of long-term retention. The first is that, for list lengths in excess of memory span, mean recall is always less than the number of items presented. The second is that the number of intrusions is very small. Since the words used in the lists are usually familiar ones and are clear instances of the categories employed, a plausible interpretation of these findings is that at recall subjects, while able to produce enough words to match list length, are reluctant to do so when they are uncertain as to the list membership of the words which occur to them (Cofer, 1967, Experiment 2). If this interpretation is valid and if, with increases in retention interval knowledge of list membership becomes less certain, then the number of intrusions should increase. The present experiment was designed to test this derivation which, of course, was confirmed in Brand's data as indicated above. Two additional factors were manipulated, and both could be related to number of intrusions. One is manner of presentation, with the list members presented either in random order or blocked by category. The other is the frequency with which the list items are given in response to the category names in an association test. The retention intervals studied were 7,14 , and 28 days following presentation and immediate recall of the list.

\section{METHOD}

The experiment followed a 2 by 2 by 3 design, with the conditions being kind of list, presentation orders, and retention intervals.

\section{Lists}

There were two lists of 40 words each, comprised in one case of the 10 highest frequency associates to the category names, occupations, weapons, four-legged animals, and articles of clothing (Cohen, Bousfield, \& Whitmarsh, 1957), and in the other case of 10 low-frequency associates of the same category name (see Cofer, Bruce, \& Reicher, 1966). The presentation conditions were blocked, in which all the items from one category were presented in sequence, then the items from another category, etc., and random, in which a nonsystematic order was employed, with the restrictions that items from each category were spread evenly through the list and that items from the same category did not appear in adjacent positions.

\section{Subjects}

There were 132 volunteers from classes in introductory psychology at the University of Maryland, tested in small groups independent of their class meetings; assignment of groups to conditions was made randomly.

\section{Procedure}

Subjects were told that they would be shown a long list of words which, after presentation, they were to recall in writing in any order in which the words occurred to them. The items were typed in capital letters and were shown for $4.4 \mathrm{sec}$ each by means of a Kodak Carousel projector. After immediate recall, the subjects were asked to return to the laboratory to participate in another experiment. When they returned for the second session, the subjects were reminded that they had seen a list earlier and were asked to recall it again.

\section{Scoring}

Scoring was for number of words correctly recalled, for categorial and other intrusions, and for two measures of clustering: (a) number of repetitions, defined as the occurrence together in recall of two items from the same category and 
(b) the ratio of repetition (RR), calculated by dividing the number of repetitions by the number of words recalled minus one.

\section{RESULTS}

\section{Immediate Recall}

The delayed recall groups did not differ in any respect at immediate recall $(\mathrm{F}<1.00)$, but manner of presentation and frequency of association were significant factors. Mean recall scores for blocked, random, high frequency, and low frequency lists were, respectively, 25.67, 22.53, 27.38, and 20.82. Categorial intrusions were more frequent than irrelevant intrusions (64 to 7) and were more frequent for the low frequency than for the high frequency list and for blocked than for random presentation. Both number of repetitions and RR scores are higher for high frequency lists and blocked presentation than for the low frequency lists and random presentation. These findings replicate those of Cofer et al., 1966).

\section{Delayed Recall}

The effects of method of presentation and frequency of association in delayed recall were comparable to those found in immediate recall, except that block presentation produced an even larger number of categorial intrusions than random presentation (and at a higher significance level) and that RR is not affected by method of presentation unless categorial intrusions are counted.

List items. The average number of list items recalled in each delay condition is shown in Table 1 . These means are lower than the overall average of 24.10 obtained in original recall indicating a decline over retention intervals. The delay effect is reliable $[F(2,120)$ $=6.34, \mathrm{p}<.005)$ although the difference between the $14-$ and 28-day groups is not significant. No other effects were significant.

Categorial intrusions. The total number of categorial intrusions (270) was much greater than the number of irrelevant intrusions (30) and the categorial intrusions provided $90 \%$ of all intrusions, as they did at immediate recall. As the data in Table 1 indicate the delay effect was significant $(p<.025)$ with the number of categorial intrusions increasing as the retention interval increased.

In view of the increase in number of intrusions over time an additional analysis was performed on list items recalled plus categorial intrusions. As expected the effects of frequency of association $[F(1,120)=65.07)$, and method of presentation $(1,120)=26.95)$, were highly significant $(\mathrm{p}<.001)$. The delay effect was also significant $[F(2,120)=3.70, p<.05]$. However, a further breakdown of this effect showed that only the quadratic component was significant $[F(1,120)=7.21$, $\mathrm{p}<.01]$, accounting for $97 \%$ of the variance. No other effects were significant.

Repetitions. The data in Table 1 also indicate that more repetitions occur in the 7-day condition than in
Table 1

Means and F Ratios for List Items (LI), Categorical Intrusions (CI), Repetitions (R), and Ratio of Repetitions $(R R)$ in Delay Conditions

\begin{tabular}{lrrrr}
\hline & \multicolumn{4}{c}{ Delay Conditions } \\
& 7 & \multicolumn{1}{c}{14} & \multicolumn{1}{c}{28} & \multicolumn{1}{c}{ F } \\
\hline LI & 19.50 & 16.14 & 15.89 & 6.34 \\
CI & 1.41 & 1.84 & 2.84 & 4.68 \\
R & 12.60 & 9.68 & 9.70 & 3.88 \\
R $^{*}$ & 13.18 & 11.05 & 11.57 & 2.11 \\
RR & .56 & .53 & .49 & 1.68 \\
RR $^{*}$ & .62 & .61 & .60 & .13 \\
\hline
\end{tabular}

${ }^{*} C I$ included as category instances.

the other delay conditions. However, the delay affect attains significance $(p<.025)$ only when intrusions are not counted as category items. The delay by frequency of association interaction was not significant in either of the delay analyses $(F s=1.28$ and 1.57$)$ contrary to the findings in original recall.

Ratio of repetition. Other than the previously noted effects of method of presentation and frequency of association no other factors were significant. The delay factor is of little consequence for RR whether or not intrusions are counted as category items. In addition, neither the Frequency by Delay interaction nor the Method of Presentation by Frequency interaction-both of which were significant in original recall-approaches significance in delayed recall.

\section{DISCUSSION}

With delay, the major interest in the present study, there is a clear decline in recall of list items but not after 14 days.

Item recall declines from original to delayed recall by 13.85 items $(29 \%)$ but categorial intrusions increase by 3.12 items (318\%). As the data in Table 1 indicate, the trend is for more categorial intrusions as the delay interval is increased. This finding accords with the expectation that knowledge of list membership recede over time, so that the subject is less accurate in his editing of responses for list membership. The result of this inaccuracy is that there is no significant difference in the number of appropriate category items (list items plus categorial intrusions) in the 7-day (20.91) and 28-day (18.73) conditions despite a significant difference between these conditions in list items recalled (19.50 vs. 15.89). A similar pattern exists with respect to number of repetitions but when ratio of repetitions is considered there is no effect of delay beyond the 7-day test. That is, there is relatively little loss of list items over time and almost no change in the relative organization of the list.

The somewhat better performance for the HF block presentation subjects in delayed recall could be attributed to either the "priming" of interitem associations during block presentation or to the encoding of category labels while the category instances are still in immediate memory. Whatever the basis, block presentation leads to better recall of list items as well as more category intrusions.

In contrast, delayed recall on LF lists is poorer. This can be attributed to the lower strength relation between category labels and category instances with LF lists. It is simply more difficult to generate these low frequnecy instances of a category when the more probable, high frequency instances have to be considered first. The very process of having to reject these high frequency associates may interfere with the generation of the low frequency items or interfere with awareness of list membership for these items. For example, presentation of the low frequency 
items may provide a unit of frequency, but the occurrence of high frequency items when the subject attempts to recall the list may also do so. Hence, the subject would not be able to discriminate which items were and were not on the list, and although he makes some high frequency intrusions, his desire to be accurate means he doesn't produce as many items as he might.

\section{REFERENCES}

Brand, H. A study of the temporal changes in the organization of retention. Journal of General Psychology, 1956, 54, 243-254.
Brand, H., \& Woods, P. J. The organization of the retention of verbal material. Journal of General Psychology, 1958, 58, 55-68.

Cofer, C. N. Does conceptual organization influence the amount retained in immediate free recall. In B. Kleinmuntz (Ed.) Concepts and the structure of memory. New York: John Wiley \& Sons, 1967.

Cofer, C. N., Bruce, D. R., \& Reicher, G. M. Clustering in free recall as a function of certain methodological variations. Journal of Experimental Psy chology, 1966, 71, 858-866.

Cohen, B. H., Bousfield, G. A., \& Whitmarsh, G. A. Cultural norms for verbal items in 43 categories. Technical Report No. 22, 1947, University of Connecticut, Contract Nonr 631(00), Office of Naval Reserach.

Bulletin of the Psychonomic Society 1975, Vol. 5 (2), 129-131

\title{
The relative proximity principle and the postreinforcement pause
}

\author{
RICHARD L. SHULL and AARON J. BROWNSTEIN \\ University of North Carolina, Greensboro, North Carolina 27412
}

\begin{abstract}
According to the relative proximity principle, a response measure should vary monotonically with the relative amount of time elapsed in an interreinforcement interval. Average response rate on fixed-interval schedules does increase monotonically with relative time, but response rate is nonmonotonically related to relative elapsed time on conj FT FR1 schedules. The present paper shows that certain characteristics of the postreinforcement pause distribution are consistent with the relative proximity principle on both fixed-interval and conjunctive schedules: The probability of terminating the postreinforcement pause, given the opportunity, increases monotonically with relative time in the interreinforcement interval on both types of schedules. Thus, the control of postreinforcement pause duration by relative time in an interreinforcement interval seems to hold over a wider range of conditions than does the control over average response rate by relative proximity.
\end{abstract}

On all schedules of reinforcement reinforcers are distributed in time. The time between successive reinforcers is the interreinforcement interval. According to the relative proximity principle (Jenkins, 1970; Staddon, 1972; see also Catania \& Reynolds, 1968; Dews, 1969), the measure of the reinforced response should vary monotonically with elapsed time in the interreinforcement interval. The "relative" indicates that the response measure is more predictable from the elapsed time expressed as a proportion of the interreinforcement interval than from elapsed time expressed in absolute time units without regard to the interreinforcement interval.

The pattern of responding on fixed-interval (FI) schedules is consistent with the relative proximity principle. Average response rate is lowest at the beginning of the interval and highest at the end.

Appearing on the surface inconsistent with the relative proximity principle is the response pattern produced by a schedule having some features in common with FI schedule: the conjunctive fixed-time, fixed-ratio

This research was supported by Grant NIMH (MH21368-01). Reprints may be obtained from $\mathbf{R}$. L. Shull, Department of Psychology, University of North Carolina at Greensboro, Greensboro, North Carolina 27412 one schedule (conj FT FR 1). On FI schedules, reinforcement depends on a response after a fixed amount of time has elapsed. On a conj FT FR 1 schedule reinforcement also depends on a response and an interval of time. But, in contrast with the FI schedule, the order does not matter. Thus, the required response can occur at any time during the interreinforcement interval on the conjunctive schedule. The conj FT FR 1 schedule often produces a pause-respond-pause response pattern so that the highest response rate occurs at the middle of the interreinforcement interval instead of at the end (Morgan, 1970; Shull, 1970, 1971b; Staddon \& Frank, 1974; Zeiler, 1974; see also Barrett, 1974). The relative proximity principle has difficulty accounting for the response rates being higher at intermediate relative proximities than at later relative proximities (see Staddon, 1972, especially Pp. 242-245).

Perhaps the problem is not with the relative proximity principle but rather with average response rate as the dependent measure. If so, it might be advantageous to consider alternative response measures. One alternative is to describe responding on these schedules as a postreinforcement pause and a terminal period. Duration, or latency measures make the most sense with this description. One such measure is the latency of the 